\title{
OpenNotes, une forme de communication moderne
}

\section{Yvonne Gilli}

Dr méd., membre du Comité central de la FMH et responsable du département Numérisation et eHealth

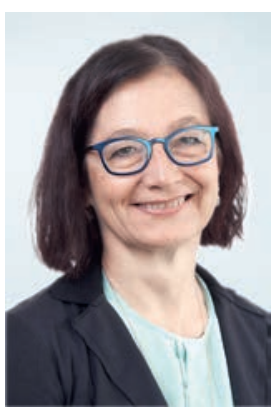

Références

1 Ziltener E, Bellinger A, Fattinger K. OpenNotes: la transparence accroît la confiance et la sécurité des patients. Bull Med Suisses. 2019;100(31-32):103336.

Byung-Chul Han, La société de la transparence, Presses Universitaires de France, 2017.

Citation de Peter Handke, A ma fenêtre le matin. Carnets du rocher 1982-1987. Verdier, 2006, p. 302.

4 Joachim Bauer,

Pourquoi je ressens ce que tu ressens, Guy Trédaniel, 2012.

5 Shanhong Luo, Effects of texting on satisfaction in romantic relationships: The role of attachment, April 2014, Computers in Human Behavior. 33:145-52.

yvonne.gilli[at]fmh.ch
La tendance aux notes en libre accès (OpenNotes) illustre parfaitement la façon dont la culture de la communication évolue avec la numérisation. Jusque dans les années 1970, les diagnostics graves n'étaient pas communiqués à la patientèle et aux proches. Aujourd'hui, le fait que les médecins et les patients aient accès rapidement aux mêmes informations spécialisées grâce aux technologies modernes est accepté. L'entretien personnel dans le cadre d'une consultation est complété numériquement, voire partiellement remplacé. Cette évolution culturelle n'est pas spécifique au corps médical. Ce qui est spécifique, c'est que l'entretien représente une part essentielle de l'art médical et que la communication dans le cadre de la relation thérapeutique entre le médecin et les patients est déterminante pour un traitement efficace. «D’abord la parole, ensuite la plante et en dernier lieu seulement la lame», cette citation est attribuée à Asclepios dans la mythologie grecque et son message central reste aujourd'hui encore d'actualité. En quoi les notes en libre accès modifient-elles la forme écrite de la prestation de relation médicale?

Les auteures de l'article «OpenNotes: la transparence accroît la confiance et la sécurité des patients» [1] paru dans cette édition du BMS proposent une bonne synthèse de l'état actuel des études et mentionnent dès le titre trois des aspects positifs clés mis en évidence dans diverses études: la transparence, la confiance et la sécurité des patients. Après que le médecin a durant des siècles été maître absolu de l'information et que les patients n'ont guère pu s'appuyer sur autre chose que leur confiance dans le traitement, il est compréhensible que la balance penche désormais en faveur d'une plus grande transparence.

Il n'existe encore que peu de documentation à l'appui des aspects négatifs de la transparence numérique. Elle pourrait pourtant être importante pour un point de vue équilibré, notamment en médecine. Byung-Chul Han [2] écrit dans son ouvrage La société de la transparence: «La distance et la pudeur ne se laissent pas intégrer dans les circuits accélérés du capital, de l'information et de la communication.» L'écrivain Peter Handke [3] opte pour une formulation plus personnelle: «Je vis de tout ce que les autres ne savent pas de moi.» Nous découvrons seulement aujourd'hui que la lecture de leur anamnèse constitue un poids pour une minorité de patients. Nous ignorons encore quelles informations le médecin ne couche plus par écrit dans la culture OpenNotes et quelles seront les répercussions de ce nouveau secret sur la qualité du traitement.

L'évolution de la culture de la communication médicale liée à la transformation numérique est complexe. Non seulement les communications orale et écrite sont différentes à bien des égards, mais la forme écrite elle-même change avec l'utilisation quotidienne des médias numériques. Lorsque nous évoquons la transparence, la confiance et la sécurité des patients, nous devons admettre que ces notions s'accompagnent d'un certain flou. C'est l'observation de la consultation médicale qui permet le mieux de décrire ce dernier [4]: «Ce sont deux personnes qui se rencontrent, dont les attitudes et les attentes conduisent à des processus intuitifs de perception et de réflexion (au sens optique) qui influencent le succès du traitement plus fortement que ne le ferait mainte mesure thérapeutique.»

Dans le cadre de l'utilisation d'OpenNotes, il sera intéressant d'étudier comment telle patiente ou tel patient utilise cette nouvelle forme de communication et s'il se dessine des parallèles avec les études en sciences sociales. Shanhong Luo [5] a étudié la relation entre la satisfaction et la fréquence des communications par SMS dans les relations amoureuses aux Etats-Unis. Les résultats ont montré que la manière d'appréhender la relation déterminait l'utilisation des messages texte. Certes, les relations thérapeutiques ne peuvent pas être exactement assimilées à des relations amoureuses. Mais les deux ont en commun de tourner autour de thèmes existentiels.

Avec leur article sur OpenNotes, les auteures [1] entament un nouveau chapitre intéressant de la communication médicale, qui sera largement influencée par les technologies numériques à l'avenir. Bon nombre de réserves exprimées par les médecins avant l'introduction d'OpenNotes sur le terrain se sont dissipées à l'usage et ont abouti à une large acceptation, qu'il convient désormais d'étudier en profondeur. 